\title{
A contribuição da visão baseada em recursos para o estudo da internacionalização: uma análise bibliométrica da produção científica entre os anos de 2007 e 2016
}

\author{
Maíra Nunes Piveta ${ }^{\mathrm{A} 1}$, Flavia Luciane Scherer ${ }^{\mathrm{A}}$, Aletéia de Moura Carpes ${ }^{\mathrm{B}}$, \\ Nathália Rigui Trindade ${ }^{A}$, Andréa Bach Rizzatti ${ }^{A}$ e Maríndia Brachak dos Santos ${ }^{A}$ \\ ${ }^{A}$ Universidade Federal de Santa Maria, UFSM, Santa Maria, RS, Brasil. \\ ${ }^{B}$ Centro Universitário Franciscana, UNIFRA, Santa Maria, RS, Brasil.
}

\section{DETALHES DO ARTIGO}

\section{Histórico do Artigo:}

Recebido: 31 de Agosto de 2017

Aceito: 17 de Novembro de 2017

Aceito: 04 de Fevereiro de 2018

Disponível online em: 01 de Maio de 2018

Sistema de revisão "Double blind review"

Editor Científico

Ilan Avrichir

\section{Palavras-chaves:}

Visão Baseada em Recursos

Internacionalização

Web of Science

Scopus

Bibliometria

\begin{abstract}
RESUMO
Este estudo buscou analisar as características das publicações sobre Visão Baseada em Recursos e Internacionalização (Resource Based View and Internationalization), nas bases de dados Web of Science e Scopus, no período de 2007 a 2016, e identificar quais tópicos estão sendo estudados junto a este tema e quais são os mais relevantes (hot topics), realizando uma comparação dos resultados encontrados nas duas bases de dados. O estudo se caracteriza como quantitativo uma vez que procurou quantificar algumas variáveis inerentes à produção científica sobre o tema pesquisado. A busca das publicações sobre VBR e Internacionalização no período de 2007 a 2016 encontrou 210 publicações na base WOS e 127 na Scopus. Tecendo uma comparação das bases pesquisadas, verificou-se que o número de publicações cresceu nos últimos dois anos e que os Estados Unidos lideram o ranking quanto ao número de publicações. Percebe-se que em ambas as bases de dados, os autores das publicações mais citadas não estão entre os autores que mais publicaram no período, com exceção de Mike Wright. Duas combinações foram classificadas como hot topics na Web of Science: Performance (desempenho) e Strategy (estratégia).
\end{abstract}

(C) 2018 Internext | ESPM. Todos os direitos reservados!

\section{INTRODUÇÃO}

As evoluções observadas no decorrer dos anos nas esferas econômicas, sociais e tecnológica impactaram significativamente a gestão organizacional, tendo a internacionalização de empresas emergido neste contexto como um importante desafio estratégico assumido pelos empreendimentos dos mais variados setores econômicos (ZEN et al., 2009). A internacionalização possibilita às empresas atuar no mercado global vislumbrando crescimento e expansão dos negócios para além das fronteiras domésticas. Ademais, o enfrentamento da acirrada concorrência imposta

\footnotetext{
${ }^{1}$ Contato do autor E-mail: mairanpiveta@gmail.com
}

pelo mercado doméstico também se configura numa das razões que motivam a internacionalização.

Destaca-se que o processo de internacionalização exige das empresas diversos fatores que as qualifiquem a atuar satisfatoriamente em mercados internacionais. Os recursos estratégicos constituemse num destes fatores. Podendo ser compreendidos à luz da teoria Resource-Based View (RBV), em português, visão baseada em recursos, os mesmos pressupõem vantagens competitivas para as empresas. De acordo com Newbert (2007), a RBV configura-se numa das teorias mais amplamente aceitas na perspectiva da gestão estratégica. isto pois 
a RBV contribui com o processo de internacionalização principalmente no que tange à dinâmica dos modos de entrada no mercado externo, à performance superior, à função dos recursos tangíveis e intangíveis da empresa do país anfitrião e, ainda, ao desenvolvimento de capacidades no decorrer do processo de internacionalização (Salazar et al., 2011).

A perspectiva teórica da Visão Baseada em Recursos (do inglês, Resource Based View - RBV) perpassa diversas temáticas sendo amplamente adotada por diversos pesquisadores em seus campos de estudo. O desenvolvimento da teoria é conferido ao trabalho seminal da economista Edith T. Penrose intitulado "The theory of the growth of the firm", publicado em 1959. Sendo a referência principal da base teórica, Penrose (1959) tratou dos aspectos internos da firma considerando a organização como um conjunto de recursos a serem utilizados. A abordagem teórica da RBV continuou a ser utilizada e desenvolvida na década de 1980 pelos autores Wernerfelt (1984) e Barney (1986). Em seguida, apresentou considerável avanço por meio de estudos de diversos pesquisadores, tais como, Prahalad e Hamel (1990), Barney (1991), Grant (1991), Peteraf (1993), Hansen et al. (2004), Newbert $(2007,2008)$ e Kraaijenbrink et al. (2010).

A relação da RBV com o processo de internacionalização de empresas se constitui mediante as contribuições relevantes que a teoria fornece no entendimento deste processo. Considerando-se a importância da teoria da RBV para o campo de estudos da administração estratégica, evidencia-se a pertinência da perspectiva teórica dos recursos para a análise do processo de internacionalização (Salazar et al., 2011). Muitos trabalhos (Peng, 2001; Fahy, 2002; Dhanaraj E Beamish, 2003; Knight e Kim, 2009), assim como os levantados no presente estudo bibliométrico, explicitam este aporte (Zen et al., 2014). De modo geral, ao decidir internacionalizarem-se, as empresas precisam contar com uma série de recursos que possibilitem o sucesso deste processo. Alguns autores contribuem neste sentido, Fahy (2002), por exemplo, dedica-se a expor uma relação de interconexões referentes às contribuições da teoria para os estudos em negócios internacionais preconizando a utilização de recursos específicos na criação de vantagem competitiva sustentável impulsionando o desempenho organizacional em ambientes globais.
Sharma e Erramilli (2004) utilizam a perspectiva teórica da RBV em seu trabalho buscando elucidar as decisões referentes ao modo de entrada no mercado externo adotado pelas organizações. Além disso, Peng (2001) apresenta as evoluções das pesquisas referentes aos negócios internacionais sob a ótica da visão baseada em recursos explicitando a ampla base de conhecimento existente e a conexão entre os assuntos. Por fim, tem-se que a RBV se tornou uma perspectiva influente e bastante utilizada nas pesquisas em negócios internacionais (Peng, 2001; Westhead et al., 2001; Hitt et al., 2006).

Assim, ao considerar que o conhecimento do estado da arte de uma área de estudo é importante para a caracterização da produção intelectual de um campo teórico (Favoreto, Amâncio-Vieira; Shimada, 2014) este estudo busca ampliar e, em especial, atualizar o conhecimento acerca das publicações que permeiam a temática da RBV e da Internacionalização. Com isto, define-se o objetivo geral do presente estudo como: analisar as características das publicações sobre Visão Baseada em Recursos e Internacionalização nas bases de dados Web of Science (WOS) e Scopus. Apresenta, ainda, como objetivos específicos, identificar os hot topics relacionados à Visão Baseada em Recursos e Internacionalização, e identificar os principais artigos citados explicitando as características fundamentais dos mesmos.

Para isto, desenvolveu-se um estudo bibliométrico, tendo em vista que de acordo com Chueke e Amatucci (2015, p.2) os estudos bibliométricos têm muito a contribuir com as pesquisas acadêmicas uma vez que estes sintetizam pesquisas e conhecimentos construídos anteriormente sobre determinado campo de saber, avançando a geração de novos conhecimentos a partir da indicação de problemas interessantes de serem investigados em pesquisas futuras, podendo "servir como base para estudos comparados, cada vez mais demandados num mundo considerado global". Desta forma, este trabalho encontra-se estruturado em quatro seções além desta introdução. A primeira apresenta o aporte teórico acerca da bibliometria e os procedimentos metodológicos adotados neste estudo. Na sequência, são elucidadas a apresentação e discussão dos resultados. E, na quarta e última seção as conclusões da pesquisa são abordadas. 


\section{BIBLIOMETRIA:}

\section{PROCEDIMENTOS METODOLÓGICOS}

O referido estudo foi desenvolvido na perspectiva de uma pesquisa bibliométrica. De acordo com Araújo (2006), a bibliometria consiste em uma técnica quantitativa e estatística de medição dos índices de produção e disseminação de conhecimento científico vislumbrando a análise quantitativa de informações bibliográficas. A utilização desta abordagem justificase pelo fato de que se desenvolveu a pesquisa com o objetivo de ampliar o conhecimento referente às publicações relacionadas à Visão Baseada em Recursos e a Internacionalização de empresas, a partir do levantamento de artigos que versam sobre o assunto. Ademais, o estudo procurou identificar quais tópicos relacionados com a temática estão sendo estudados e quais são os temas em voga.

Além disto, conforme Vanti (2002) a bibliometria auxilia a identificar tendências de crescimento do conhecimento em uma área, identificar revistas de uma disciplina, verificar tendências de publicação, mapear a produtividade de autores, organizações e países, medir o crescimento de determinadas áreas e o surgimento de novos temas, entre outros aspectos. Neste contexto os métodos bibliométricos são quantitativos, pois buscam medir e quantificar algumas variáveis referentes à produção científica (CRONIN, 2001), que no caso deste estudo, concernem a Visão Baseada em Recursos e Internacionalização ("Resource based view" and "Internationalization").

Neste contexto, a obtenção dos dados e informações necessárias ao estudo foram coletados por intermédio das bases de dados Web of Science do Institute for Scientific Information (ISI) e Scopus da Elsevier. A escolha se deu mediante o notório reconhecimento que as bases apresentam no cenário mundial acadêmico e nos demais estudos de caráter bibliométrico. A WOS (Web of Science) consiste em uma base multidisciplinar que indexa apenas os periódicos mais citados em suas respectivas áreas. Ademais, constitui-se num índice de citações na web, que além de possibilitar a identificação das citações recebidas, referências utilizadas e registros relacionados, disponibiliza ferramentas para análise de citações, referências, índice $h$, permitindo análises bibliométricas. Possui atualmente cerca de 12.000 periódicos indexados (CAPES, 2017).

Já a Scopus consiste numa base de dados das áreas de Ciências Biológicas, Ciências da Saúde,
Ciências Físicas e Ciências Sociais. A base indexa títulos acadêmicos revisados por pares, títulos de acesso livre, anais de conferências, publicações comerciais, séries de livros, páginas web de conteúdo científico e patentes de escritórios. Funcionalidades de apoio à análise dos resultados como, por exemplo, identificação de autores e filiações, análises de citações, de publicações e índice $h$ são disponibilizados ao usuário (CAPES, 2017).

Assim, os esforços desta pesquisa se concentraram no rastreamento e descrição de indicadores no que se refere a produção acadêmica sobre a temática abordada. Para Sancho (2002), os indicadores bibliométricos são ferramentas de avaliação e podem ser de quatro tipo, a saber: i) indicadores de qualidade científica; ii) indicadores de atividade científica; iii) indicadores de impacto científico e iv) indicadores de associações temáticas. Neste estudo, optou-se por dar ênfase aos indicadores de atividade científica uma vez que estes vislumbram quantificar a atividade científica desenvolvida, principalmente no que tange ao cálculo do número de publicações de determinados grupos, instituições ou países e suas distribuição, à produtividade dos autores e à colaboração nas publicações (Sancho, 2002).

A partir disto, objetivando a viabilização da análise bibliométrica e utilizando-se dos recursos de análise disponibilizados nas bases de dados WOS e Scopus, estabeleceu-se doze características a serem analisadas, conforme demonstra a Tabela 1. Vale destacar que estas características foram definidas com base nos indicadores de atividade científica (Sancho, 2002) e em diversos estudos bibliométricos que fazem uso destes aspectos (Carpes et al., 2011; Rocha et al., 2013; Avila et al., 2014; Motke; Ravanello; Rodrigues, 2016).

Tab. 1

Modelo conceitual para análise bibliométrica

\begin{tabular}{lcc}
\hline $\begin{array}{l}\text { Características gerais } \\
\text { das publicações }\end{array}$ & $\begin{array}{c}\text { Web of Science } \\
(\mathbf{2 0 0 7}-\mathbf{2 0 1 6 )}\end{array}$ & $\begin{array}{c}\text { Scopus } \\
(\mathbf{2 0 0 7}-\mathbf{2 0 1 6 )}\end{array}$ \\
\hline Total de publicações & $\mathrm{X}$ & $\mathrm{X}$ \\
Áreas temáticas & $\mathrm{X}$ & $\mathrm{X}$ \\
Tipos de documentos & $\mathrm{X}$ & $\mathrm{X}$ \\
Anos das publicações & $\mathrm{X}$ & $\mathrm{X}$ \\
Autores & $\mathrm{X}$ & $\mathrm{X}$ \\
Fontes das publicações & $\mathrm{X}$ & $\mathrm{X}$ \\
Instituições & $\mathrm{X}$ & $\mathrm{X}$ \\
Países & $\mathrm{X}$ & $\mathrm{X}$ \\
Idiomas & $\mathrm{X}$ & \\
Índice $h-b$ & $\mathrm{X}$ & $\mathrm{X}$ \\
Índice $m$ & $\mathrm{X}$ & $\mathrm{X}$ \\
Autores versus citações & $\mathrm{X}$
\end{tabular}

Fonte: Elaborado pelas autoras 
Como pode-se perceber, a partir da Tabela 1, além de englobar os indicadores de atividade científica (Sancho, 2002), também buscou-se analisar alguns indicadores de impacto científico, aqui compreendidos nos índices h-b e m, que serviram para posterior realização e análise de hot topics. Conforme Hirsch (2005), faz-se necessária a quantificação do impacto e relevância da produção científica individual com o intuito de se obter a análise de pesquisadores e a comparação de propósitos. Destarte, Hirsch (2005) propõe um número único o h-index (índice-h) como uma forma acessível e útil para caracterizar a produção científica de um pesquisador. Posteriormente, Banks (2006) contribuiu com o índice $h-b$, uma extensão do hindex, onde as citações são listadas em ordem decrescente e é alcançado a partir do número de citações de um tópico ou combinação em determinado período. Este índice é encontrado em publicações que tenham obtido um número de citações igual ou maior à sua posição no ranking (Banks, 2006).

Banks (2006) explica ainda como se calcula o índice $m$, o qual é alcançado por meio da divisão do índice $\mathrm{h}$-b pelo período de anos que se deseja obter informações ( $n$ ). Para a análise dos índices h-b e m, foram utilizadas as definições de Banks (2006) expostas no Tabela 2. Destaca-se que, a partir das definições de Banks (2006) expostas anteriormente, neste estudo serão considerados hot topics as combinações com índice $m \geq 2$. Ainda, na sequência serão melhor explicados os procedimentos adotados para realização desta análise.

Tab. 2

Definições para classificação de hot topics

\begin{tabular}{ll}
\hline Índice $\mathrm{m}$ & Tópico/combinação \\
\hline $0<m \leq 0,5$ & $\begin{array}{l}\text { Pode ser de interesse para pesquisadores em um } \\
\text { campo específico de pesquisa, o qual engloba uma } \\
\text { comunidade pequena. }\end{array}$ \\
\hline $0,5<\mathrm{m} \leq 2$ & $\begin{array}{l}\text { Provavelmente pode ser um "hot topic" como área } \\
\text { de pesquisa, no qual a comunidade é muito grande } \\
\text { ou o tópico/combinação apresenta características } \\
\text { muito interessantes }\end{array}$ \\
\hline $\mathrm{m} \geq 2$ & $\begin{array}{l}\text { É considerado um "hot topic", tópico exclusivo com } \\
\text { alcance não apenas na sua própria área de pesquisa e } \\
\text { é provável que tenha efeitos de aplicação ou } \\
\text { características únicas }\end{array}$ \\
\hline
\end{tabular}

Fonte: Banks (2006).

Por conseguinte, a coleta dos dados foi realizada a partir dos mecanismos de busca da WOS e da Scopus. Em um primeiro momento digitaram-se as palavras: Visão baseada em recursos e Internacionalização ("Resource based view" and Internationalization) como tópico no campo de pesquisa das bases WOS e Scopus, no idioma inglês, delimitando-se o período temporal que compreende os anos de 2006 a 2017. Cabe destacar que, as referidas palavras-chave foram utilizadas uma vez que ao realizar testes e a partir de uma leitura prévia dos artigos mais citados nos resultados da busca, esta combinação foi a que mais se aproximou do interesse do escopo definido para o presente trabalho. Ainda, no que tange a delimitação do período de 10 anos, destaca-se que esta limitação temporal foi realizada buscando-se apresentar o nível mais contemporâneo (atual) de conhecimento observado neste campo de estudo. O que é corroborado na pesquisa de Moraes, Strehlau e Turolla (2015) que também adotam este período baseados em diversos autores e estudos bibliométricos.

Em um segundo momento, realizou-se o levantamento dos hot topics para averiguar os tópicos mais relevantes de estudo no que tange a temática. Para isso, inicialmente foram identificados os tópicos a serem combinados com "Resource based view" e Internationalization. Estes tópicos foram selecionados a partir do levantamento das palavraschave dos 10 artigos mais citados em cada uma das bases. Com isto, foram selecionadas as 20 palavraschave mais citadas em cada base, aqui denominadas tópicos.

Tab. 3

Etapas da pesquisa

\begin{tabular}{|c|c|}
\hline $\begin{array}{l}\text { Etapas da } \\
\text { Pesquisa }\end{array}$ & Descrição \\
\hline Primeira & $\begin{array}{l}\text { Pesquisa do tópico "resourced based view" e } \\
\text { internationalization na WOS e Scopus; }\end{array}$ \\
\hline & Análise das característi cas das publicações; \\
\hline \multirow[t]{2}{*}{ Segunda } & $\begin{array}{l}\text { Identificação dos tópicos relacionados a Visão } \\
\text { baseada em recursos e Internacionalização; }\end{array}$ \\
\hline & Seleção de 20 tópicos - WOS e Scopus; \\
\hline Terceira & $\begin{array}{l}\text { Combinação na WOS e Scopus dos tópicos } \\
\text { iniciais com os } 20 \text { tópicos relacionados; }\end{array}$ \\
\hline Quarta & $\begin{array}{l}\text { Cálculo índice h-b e m e identificação dos hot } \\
\text { topics na WOS e Scopus; }\end{array}$ \\
\hline Quinta & $\begin{array}{l}\text { Relação entre autores com mais publicações e } \\
\text { publicações mais cotadas na WOS e Scopus; }\end{array}$ \\
\hline
\end{tabular}

Fonte: Elaborado pelas autoras

A partir disto, nos mecanismos de busca de cada uma das bases de dados, realizou-se a combinação de cada um dos 20 tópicos selecionados com os tópicos iniciais da pesquisa ("Resource based view" and Internationalization) no período de, também, 10 anos (2006 a 2017) para levantamento do índice h-b de cada um deles. Na sequência, então, os "hot topics" 
foram elencados por meio do cálculo do índice $m$ (divisão do índice h-b pelo período de anos que se deseja obter informações, neste caso 10 anos), conforme definido por Banks (2006).

Por fim, a Tabela 3 a seguir evidencia as etapas para realização da pesquisa.

Dado o exposto, de acordo com as etapas apresentadas na Tabela 3 foi realizada a análise bibliométrica do referido estudo, cujos resultados são apresentados na seção a seguir.

\section{APRESENTAÇÃO E DISCUSSÃO DOS RESULTADOS}

A consolidação da globalização combinada com o avanço das tecnologias de informação e comunicação tornaram a internacionalização de empresas uma constante. A temática é um fenômeno que está em voga nas pesquisas acadêmicas, destacando-se, por seminais, os estudos de Vernon (1966), Johanson e Weindersheim-Paul (1975) e Dunning (1980). De acordo com Hitt et al. (2006), a maioria das pesquisas se concentra na elucidação das motivações para as empresas buscarem mercados externos deixando uma grande lacuna sobre quais são os recursos específicos que as empresas precisam para prosperar com êxito nos mercados internacionais. O presente estudo coaduna com o exposto mediante a intenção de analisar a contribuição da Visão Baseada em Recursos para o estudo da internacionalização das organizações.

Os resultados da pesquisa evidenciaram as principais características da produção científica relacionada às temáticas "Resource based view" and Internationalization. Primeiramente foi realizada a pesquisa com os termos na base WOS, no período de 2007 a 2016, sendo encontradas 210 publicações. Num segundo momento, procedeu-se com a pesquisa na base de dados da Scopus, sendo encontradas no mesmo período 127 publicações. Após, realizou-se a comparação entre o número de publicações por autor e o número de citações.

\subsection{Características Gerais das publicações sobre Visão Baseada em Recursos e Internacionalização na Web of Science e Scopus}

A seguir encontram-se dispostas as características gerais das publicações relacionadas ao tema de acordo com as seguintes categorias: áreas temáticas, tipo de documentos, ano das publicações, autores, título das fontes, instituições, países e idiomas, conforme exposto previamente na Tabela 1.

Tab. 4

Principais áreas temáticas

\begin{tabular}{|c|c|c|c|c|c|}
\hline Web of Science & & & Scopus & & \\
\hline Áreas Temáticas & $\begin{array}{c}\text { № } \\
\text { public. }\end{array}$ & $\begin{array}{c}\% \text { de } \\
210\end{array}$ & Áreas Temáticas & $\begin{array}{l}\text { № } \\
\text { public. }\end{array}$ & $\begin{array}{l}\% \text { de } \\
127\end{array}$ \\
\hline Business Economics (Economia) & 197 & 93,8 & $\begin{array}{l}\text { Business, Management and Accounting } \\
\text { (Negócios, Gestão e Contabilidade) }\end{array}$ & 118 & 92,9 \\
\hline Engineering (Engenharia) & 9 & 4,2 & $\begin{array}{l}\text { Economics, Econometrics and Finance } \\
\text { (Economia, econometria e finanças) }\end{array}$ & 52 & 40,9 \\
\hline $\begin{array}{l}\text { Social Sciences Other Topics } \\
\text { (Ciências Sociais e Outros Tópicos) }\end{array}$ & 8 & 3,8 & Social Sciences (Ciências Sociais) & 17 & 13,4 \\
\hline $\begin{array}{l}\text { Operations Research Management Science } \\
\text { (Ciência de gerenciamento de operações de } \\
\text { pesquisa) }\end{array}$ & 7 & 3,3 & Decision Sciences (Ciências da decisão) & 9 & 7,1 \\
\hline Public Administration (Administração Pública) & 5 & 2,3 & Engineering (Engenharia) & 6 & 4,7 \\
\hline $\begin{array}{l}\text { Science Technology Other Topics } \\
\text { (Ciência tecnologia outros tópicos) }\end{array}$ & 4 & 1,9 & $\begin{array}{l}\text { Arts and Humanities } \\
\text { (Artes e humanidades) }\end{array}$ & 4 & 3,1 \\
\hline Agriculture (Agricultura) & 3 & 1,4 & $\begin{array}{l}\text { Computer Science } \\
\text { (Ciência da Computação) }\end{array}$ & 3 & 2,4 \\
\hline Computer Science (Ciência da Computação) & 3 & 1,4 & $\begin{array}{l}\text { Environmental Science } \\
\text { (Ciência Ambiental) }\end{array}$ & 3 & 2,4 \\
\hline $\begin{array}{l}\text { Environmental Sciences Ecology } \\
\text { (Ciência Ambiental e Ecologia) }\end{array}$ & 3 & 1,4 & $\begin{array}{l}\text { Agricultural and Biological Sciences } \\
\text { (Agricultura e Ciências Biológicas) }\end{array}$ & 2 & 1,6 \\
\hline Education Educational Research & 2 & 0,9 & Energy (Energia) & 1 & 0,8 \\
\hline (Formação acadêmica pesquisa educacional) & & & Mathematics (Matemática) & 1 & 0,8 \\
\hline
\end{tabular}

Fonte: Web of Science e Scopus (Fev./2017) 
Tab. 5

Tipos de Documentos

\begin{tabular}{|c|c|c|c|c|c|}
\hline Web of Science & & & Scopus & & \\
\hline Tipos de Documentos & Frequência & $\%$ de 210 & Tipos de Documentos & Frequência & \% de 127 \\
\hline \multirow[t]{2}{*}{ Article } & 179 & 85,2 & Article & 104 & 81,9 \\
\hline & & & Review & 10 & 7,9 \\
\hline \multirow[t]{2}{*}{ Proceedings Paper } & 18 & 8,5 & Article in Press & 7 & 5,5 \\
\hline & & & Book Chapter & 2 & 1,6 \\
\hline \multirow[t]{2}{*}{ Review } & 12 & 5,7 & Conference Paper & 2 & 1,6 \\
\hline & & & Editorial & 1 & 0,8 \\
\hline Editorial Material & 3 & 1,4 & Note & 1 & 0,8 \\
\hline
\end{tabular}

Fonte: Web of Science e Scopus (Fev./2017)

\section{Áreas Temáticas}

Iniciando-se as análises, o primeiro aspecto categórico a ser averiguado são as áreas temáticas. Sendo assim, as dez principais áreas temáticas encontradas nas bases podem ser conferidas na Tabela 4.

A diversidade quanto às áreas temáticas relacionadas com a temática Resource based view and Internationalization, pode ser averiguada por meio da visualização da Tabela 4. Foi possível, ainda, notar certa similaridade no que tange às áreas temáticas de ambas as bases. Na WOS a área temática Business Economics (Economia) merece destaque apresentando um número de 197 publicações.

Já no que se refere a Scopus, a área Business, Management and Accounting (Negócios, Gestão e Contabilidade) destacou-se com a incidência de 118 publicações. Cabe destacar que a base WOS apresentou um número maior de publicações quando comparado com a Scopus.

\section{Tipos de Documentos}

A Tabela 5 apresenta os tipos de documentos referentes às publicações encontradas na WOS e na Scopus.

Por meio da análise da Tabela 5 acima pode-se notar que em ambas as bases de dados a maioria das publicações encontradas são artigos, correspondendo a $85,2 \%$ das publicações na WOS e $81,9 \%$ na Scopus. Os demais tipos de documentos elencados não se destacaram de forma expressiva, mas as suas frequências estão demonstradas na Tabela 5. O predomínio de artigos nas bases reflete as características dos periódicos que estão nelas incluídos e evidencia trabalhos mais maduros se comparados a proceedings papers, por exemplo.

\section{Principais Anos de Publicação}

A Tabela 6 aborda a relação de quantidade de documentos no que tange à distribuição entre os anos cujo período a presente pesquisa se propôs a pesquisar.

Na base WOS observou-se que o ano com maior destaque em termos de número de publicações foi o de 2016 com 37 publicações representando 17,6\% do total. Já na Scopus, o ano de 2014 destacou-se apresentando 23 publicações $(18,1 \%)$ seguido pelo ano de 2016, anteriormente mencionado em destaque na WOS, com 22 publicações (17,3\%).

$\mathrm{Na}$ Figura 1, a seguir, a distribuição das publicações no decorrer dos anos pesquisados apresenta-se disposta num gráfico que busca traçar um comparativo dos resultados encontrados em ambas as bases de modo a facilitar a visualização.

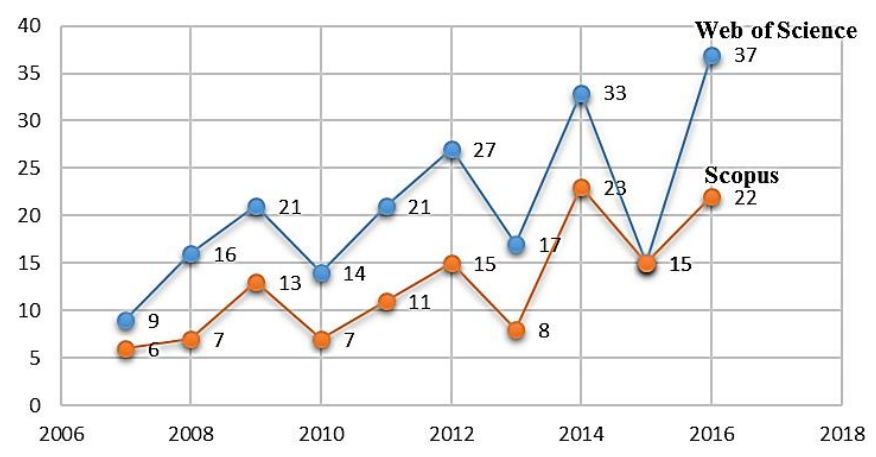

Fig. 1

Anos de Publicação

Fonte: Elaborado pelas autoras

Por meio da Figura 1 pode-se perceber que as publicações acerca da "Resourced based view" and Internationalization nas bases WOS e Scopus não apresentaram um comportamento de crescimento estável durante o período determinado para a pesquisa (2007 - 2016). No entanto, pode-se notar que nos últimos dois anos houve um salto no número 
Tab. 6

Principais anos de publicação

\begin{tabular}{ccc|ccc}
\hline & Web of Science & \multicolumn{3}{c}{ Scopus } \\
\hline Anos de Publicação & No public. & \% de 210 & Anos de Publicação & № public. & \% de 127 \\
\hline 2016 & 37 & 17,6 & 2016 & 22 & 17,3 \\
2015 & 15 & 7,1 & 2015 & 23 & 11,8 \\
2014 & 33 & 15,7 & 2014 & 8 & 18,1 \\
2013 & 17 & 8,0 & 2013 & 15 & 6,2 \\
2012 & 27 & 12,8 & 2012 & 11 & 71,8 \\
2011 & 21 & 10 & 2011 & 13 & 8,6 \\
2010 & 14 & 6,6 & 2010 & 7 & 5,5 \\
2009 & 21 & 10 & 2009 & 6 & 10,2 \\
2008 & 16 & 7,6 & 2007 & & 5,5 \\
2007 & 9 & 4,2 & & & 4,7 \\
\hline
\end{tabular}

Fonte: Web of Science e Scopus (Fev./2017)

de publicações. Embora haja queda em anos específicos, como 2015, a tendência geral é de crescimento no número de artigos, saindo de 9 em 2007 e chegando em 37 em 2016 na Web of Science e evoluindo de 6 para 22 ao longo do período analisado na Scopus. Os anos de 2013 e 2015 mostram, em ambas as bases, quedas significativas no número de artigos, o que é curioso, mas que pode evidenciar certo comportamento isomórfico entre os pesquisadores e seus interesses de estudo.

$\mathrm{Na}$ sequência, os principais autores elencados junto às bases serão apresentados.

\section{Principais Autores}

Os autores que mais publicaram sobre a temática Visão Baseada em Recursos e Internacionalização, nas duas bases analisadas, estão dispostos na Tabela 7 a seguir. Ressalte-se que foram selecionados apenas os oito primeiros para fins desta apresentação.

Por meio da análise do quadro tornou-se possível verificar uma grande diversidade e multiplicidade quanto à autoria dos trabalhos, já que não existe um destaque com relação à quantidade de trabalhos publicados acerca da temática sendo atribuído a determinado autor. Na base WOS o destaque foi do autor Mike Wright apresentando cinco artigos publicados. Mike é professor na escola de negócios do Imperial College London desde setembro de 2011 atuando na área de empreendedorismo. Na base Scopus o destaque com 3 três publicações foi para os autores Kumar, V. (Professor e Diretor do Programa de Marketing do J. Mack Robinson College of Business da Georgia State University em Atlanta, EUA) e Rajshekhar G Javalgi (Professor de Marketing e Negócios Internacionais na Cleveland State University, EUA).

A não concentração de artigos em poucos autores pode ser um fator positivo, pois evidencia que o tema encontra interesse em diferentes grupos de pesquisadores, oriundos de diferentes escolas. Como resultado, pode-se ter uma diversidade de olhares embebidos de traços culturais díspares - que pode contribuir para maior riqueza metodológica e conceitual.

Tab. 7

Principais autores

\begin{tabular}{lcclcc}
\hline Web of Science & \multicolumn{3}{l}{ Scopus } \\
\hline Autores & Artigos publicados & \% de 210 & Autores & Artigos publicados & $\%$ de 127 \\
Wright, M. & 5 & 2,3 & Kumar, V. & 3 & 2,3 \\
Baum, M. & 3 & 1,4 & Javalgi, R. G. & 3 & 2,3 \\
Buckley, P. J. & 3 & 1,4 & Bianchi, C. & 2 & 1,5 \\
Kabst, R. & 3 & 1,4 & Che Senik, Z. & 2 & 1,5 \\
Raymond, L. & 3 & 1,4 & Edelman, L. F. & 2 & 1,5 \\
Ruzzier, M. & 3 & 1,4 & Haapanen, L. & 2 & 1,5 \\
Schwens, C. & 3 & 1,4 & Hauser, C. & 2 & 1,5 \\
St-Pierre, J. & 3 & 1,4 & Manolova, T. S. & 2 & 1,5 \\
\hline
\end{tabular}

Fonte: Web of Science e Scopus (Fev./2017) 
Tab. 8

Principais fontes de publicação

\begin{tabular}{lcclcc}
\hline Web of Science & \multicolumn{5}{l}{ Scopus } \\
\hline Fontes de Publicação & \# Art & \% de & Fontes de Publicação & $\#$ & $\%$ de \\
& 210 & & & 127 \\
International Business Review & 23 & 10,9 & Journal of World Business & 8 & 6,2 \\
Journal of World Business & 13 & 6,1 & International Business Review & 7 & 5,5 \\
Journal of International Business Studies & 11 & 5,2 & International Marketing Review & 6 & 4,7 \\
International Marketing Review & 9 & 4,2 & European Journal of International Management & 4 & 3,1 \\
Journal of International Management & 7 & 3,3 & Journal of Business Research & 4 \\
International Small Business Journal & 6 & 2,8 & Journal of International Business Studies & 4 \\
Journal of International Marketing & 6 & 2,8 & Multinational Business Review & 3,1 \\
\hline
\end{tabular}

Fonte: Web of Science e Scopus (Fev./2017)

\section{Principais Fontes de Publicação}

A Tabela 8 apresenta as principais fontes de publicações relacionadas com a temática Resource based view and Internationalization.

Com relação às fontes de publicação, as que mais destacaram-se na base WOS foram as seguintes: International Business Review, Journal of World Business e Journal of International Business Studies. Já na base Scopus, o destaque foi para o Journal of World Business, International Business Review e International Marketing Review.

Ressalte-se que as duas principais fontes de publicação foram as mesmas para ambas as bases. 0 periódico International Business Review figurou como o mais representativo na WOS apresentando 23 artigos e em segundo lugar na Scopus com 7 registros. Já o Journal of World Business apresentou 8 registros na Scopus e 13 na WOS figurando em primeiro e segundo lugar respectivamente nas bases mencionadas. Tem-se aí um retrato dos periódicos que mais recebem contribuições relativas aos dois temas analisados, indicando a necessidade de tê-los como referência em estudos que abordem RBV e internacionalização.

\section{Principais Instituições}

As instituições que mais publicaram trabalhos relacionados ao tema Resource based view and Internationalization estão em destaque na Tabela 9.

As instituições que mais se destacaram na WOS, foram as seguintes: University Leeds (Reino Unido); University Nottingham (Reino Unido). E, na Scopus as seguintes universidades: Cleveland State University (Estados Unidos da América); Queensland University of Technology QUT (Austrália); The University of Sydney (Austrália).

\section{Idiomas e Países de Origem}

O idioma inglês se destaca veementemente na base WOS, com 206 registros (98\%). Na base Scopus não foi possível acessar este dado. No que tange aos países de origem das publicações, a Tabela 10, a seguir, apresenta os dez principais países que possuem publicações relacionadas ao tema pesquisado.

De acordo com a análise da Tabela 10 tornou-se possível verificar que em ambas as bases os Estados Unidos da América figuram em primeiro lugar no que diz respeito ao número de publicações. Na WOS o

Tab. 9

Principais instituições

\begin{tabular}{|c|c|c|c|c|c|}
\hline Web of Science & & & Scopus & & \\
\hline Instituições & \# Art & $\%$ de 210 & Instituições & \# Art & $\%$ de 127 \\
\hline University Leeds & 7 & 3,3 & Cleveland State University & 4 & 3,1 \\
\hline University Nottingham & 7 & 3,3 & Queensland University of Technology QUT & 4 & 3,1 \\
\hline Copenhagen Business School & 4 & 1,9 & The University of Sydney & 4 & 3,1 \\
\hline Natl Chengchi University & 4 & 1,9 & Universiti Kebangsaan Malasya & 3 & 2,3 \\
\hline University Primorska & 4 & 1,9 & University of Kent & 3 & 2,3 \\
\hline \multirow[t]{4}{*}{ University Zaragoza } & 4 & 1,9 & University of Macau & 3 & 2,3 \\
\hline & & & University of Melbourne & 3 & 2,3 \\
\hline & & & St. Louis University & 3 & 2,3 \\
\hline & & & Universidade Nove de Julho & 3 & 2,3 \\
\hline
\end{tabular}

Fonte: Web of Science e Scopus (Fev./2017) 
Tab. 10

Países de origem

\begin{tabular}{lcclcc}
\hline Web of Science & \multicolumn{3}{l}{ Scopus } \\
\hline Países & Publicações & \% de 210 & Países & Publicações & $\%$ de 127 \\
Estados Unidos da América & 51 & 24,2 & Estados Unidos da América & 32 & 25,1 \\
Inglaterra & 36 & 17,1 & Reino Unido & 18 & 14,1 \\
Espanha & 25 & 11,9 & Austrália & 13 & 10,2 \\
China & 19 & 9,0 & Espanha & 13 & 10,2 \\
Austrália & 16 & 7,6 & Brasil & 9 & 7,0 \\
Alemanha & 14 & 6,6 & China & 9 & 7,0 \\
Netherlands & 12 & 5,7 & Itália & 7 & 5,5 \\
Canada & 11 & 5,2 & Indefinido & 7 & 5,5 \\
Itália & 11 & 5,2 & Alemanha & 6 & 4,7 \\
Finlândia & 9 & 4,2 & Taiwan & 6 & 4,7 \\
\hline
\end{tabular}

Fonte: Web of Science e Scopus (Fev./2017)

país apresentou 51 publicações enquanto que na Scopus foram 32 publicações.

Na sequência, na base WOS a Inglaterra apresenta 36 publicações representando 17,1\% da amostra seguida pela Espanha com 25 registros. Já na Scopus, o Reino Unido, a Austrália e a Espanha destacam-se na sequência com 18, 13 e 13 registros de publicações respectivamente. Para ambas a bases (WOS e Scopus), os demais países que figuraram entre os dez com maior número de publicações podem ser conferidos no quadro exposto acima.

Na base Scopus o Brasil figura na quinta posição, com 7,0\% de representatividade no total de artigos publicados, o que pode ser considerado um desempenho ainda tímido, embora superior a outros importantes países. Na WOS, ao contrário, o Brasil não figurou entre as 10 primeiras posições. Talvez o idioma ainda seja uma importante barreira a explicar a baixa participação de autores brasileiros dentre os que mais publicam nos temas avaliados.

\subsection{Os "Hot Topics" relacionados com a Visão Baseada em Recursos e Internacionalização}

Esta etapa da pesquisa dedicou-se a investigar os principais tópicos relacionados à Visão baseada em recursos e Internacionalização que estão sendo estudados no período que compreende os anos de 2007 e 2016. Levando-se em consideração uma análise prévia das publicações encontradas na Web of Science e Scopus, foram selecionados 20 tópicos relacionados à temática em cada base conforme a

Tab. 11

Hot topics no estudo sobre o tema pesquisado na Web of Science.

\begin{tabular}{lccc} 
Tópicos - Base WOS & Total de publicações & Índice h-b & Coeficiente $m$ \\
\hline Performance (Desempenho) & 159 & 24 & 2,4 \\
Strategy (Estratégia) & 98 & 22 & 2,2 \\
Knowledge (Conhecimento) & 89 & 17 & 1,7 \\
Foreign (Estrangeiros) & 75 & 17 & 1,7 \\
Network (Rede) & 45 & 16 & 1,6 \\
Exporting (Exportação) & 68 & 16 & 1,6 \\
Entrepreneurship (Empreendedorismo) & 37 & 14 & 1,4 \\
Innovation (Inovação) & 53 & 13 & 1,3 \\
Learning (Aprendizagem) & 28 & 12 & 1,2 \\
Emerging economies (Economias emergentes) & 25 & 12 & 1,2 \\
Dynamic capabilities (Capacidades dinâmicas) & 43 & 12 & 1,2 \\
Manufacturing (Fabricação) & 44 & 11 & 1,1 \\
Alliance (Aliança) & 12 & 9 & 0,9 \\
Globalization (Globalização) & 18 & 8 & 0,8 \\
Multinational enterprises (Empresas multinacionais) & 16 & 8 & 0,8 \\
Knowledge transfer (Transferência de conhecimento) & 11 & 5 & 0,5 \\
Small and medium-sized enterprises (Pequenas e médias empresas) & 20 & 5,5 \\
Collaboration (Colaboração) & 4 & 4 \\
Policy implications (Implicações de política) & 5 & 0,4 \\
Portfolio (Portfólio) & 7 & 2 & 0,4
\end{tabular}

Fonte: Web of Science (Fev./2017) 
conduta já apresentada na seção de número 2 "Bibliometria: Procedimentos Metodológicos".

Posteriormente, foi realizada a combinação de cada um destes tópicos com a expressão "Resource based view" and Internationalization, sendo calculado o total de publicações para cada combinação (tópico relacionado), o h-b e o coeficiente $m$, tanto na base Web of Science quanto na Scopus. Os resultados encontram-se apresentados nas Tabelas 11 e 12 a seguir.

Pautando-se nas considerações tecidas por Banks (2006) foi possível classificar como hot topic ou tópico quente, apenas duas combinações dos temas Resource based view and Internationalization que apresentaram um coeficiente $m \geq 2$ na base de dados Web of Science, são elas: Performance (desempenho) e Strategy (estratégia) com coeficientes de 2,4 e 2,2 respectivamente. Tais tópicos são amplos em si e abordam olhares que conversam diretamente com a temática de análise RBV e internacionalização, ambos fortemente vinculados a considerações estratégicas e de desempenho.

Os tópicos Network (rede), Knowledge (conhecimento), Learning (aprendizagem), Manufacturing (fabricação), Innovation (inovação), Foreign (estrangeiros), Exporting (exportação), Globalization (globalização), Alliance (aliança), Entrepreneurship (empreendedorismo), Emerging economies (economias emergentes), Knowledge transfer (transferência de conhecimento), Small and medium-sized enterprises (pequenas e médias empresas), Dynamic capabilities (capacidades dinâmicas) e Multinational enterprises (empresas multinacionais) obtiveram um coeficiente $\mathrm{m}$ entre 0,5 e 2, sendo considerados como hot topics emergentes em suas áreas de pesquisa. Como emergentes, merecem a atenção dos pesquisadores pois podem indicar tendências de interesse crescente da academia. Ademais, os tópicos que apresentaram $m \leq 0,5$ podem ser de interesse para pesquisadores em um campo específico de pesquisa.

Já na base Scopus, não foi possível encontrar nenhum hot topic para a combinação proposta. Contudo, onze tópicos obtiveram um coeficiente $m$ entre 0,5 e 2 , sendo considerados como hot topics emergentes em suas áreas de pesquisa, são eles: Performance (desempenho), Network (Rede), Size (Tamanho), Industry (Indústria), China, Institutions (Instituições), Exports (Exportações), Small and medium-sized enterprises (Pequenas e médias empresas), Small Firms (Pequenas empresas), Institutional theory (Teoria Institucional), Multinational enterprises (Empresas multinacionais). Os nove tópicos restantes que podem ser visualizados na Tabela 12 apresentaram $m \leq 0,5$ sendo classificados na mesma condição supracitada no parágrafo anterior.

Tab. 12

Hot topics no estudo sobre o tema pesquisado na Scopus.

\begin{tabular}{lccc}
\hline Tópicos - Base Scopus & Total de publicações & Índice h-b & Coeficiente $m$ \\
\hline Performance (Desempenho) & 36 & 14 & 1,4 \\
Industry (Indústria) & 34 & 11 & 1,1 \\
China & 15 & 9 & 0,9 \\
Exports (Exportações) & 20 & 9 & 0,9 \\
Network (Rede) & 20 & 7 & 0,7 \\
Small and medium-sized enterprises (Pequenas e médias empresas) & 15 & 7 & 0,7 \\
Size (Tamanho) & 12 & 6 & 0,6 \\
Small Firms (Pequenas empresas) & 10 & 6 & 0,6 \\
Institutions (Instituições) & 11 & 5 & 0,5 \\
Institutional theory (Teoria Institucional) & 8 & 5 & 0,5 \\
Multinational enterprises (Empresas multinacionais) & 11 & 5 & 0,5 \\
Firm Performance (Desempenho da firma) & 5 & 4 & 0,4 \\
Entrepreneurial firms (Empresas empreendedoras) & 6 & 4 & 0,4 \\
Structural Equation Modeling (Modelagem de equações estruturais) & 4 & 3 & 0,3 \\
Organizational learning (Aprendizado organizacional) & 3 & 2 & 0,2 \\
Japan (Japão) & 1 & 1 & 0,1 \\
Quality Management (Gestão da qualidade) & 1 & 1 & 0,1 \\
Natural Environment (Ambiente natural) & 1 & 1 \\
Biotech (Biotecnologia) & 2 & 1 \\
ISO 900O & 1 & 1 & 0,1 \\
\hline
\end{tabular}

Fonte: Web of Science (Fev./2017) x 


\subsection{Relação dos trabalhos com maior número de citações}

Nesta etapa do estudo buscou-se averiguar por meio do levantamento e apreciação das produções científicas mais citadas nas bases Web of Science e Scopus quais são os delineamentos prestados pelos autores ao tratar das temáticas "Resource based view" e Internationalization e as características fundamentais encontradas nestes estudos. Nesta fase foram selecionadas as dez publicações que obtiveram o maior número de citações em cada base, conforme está apresentado nas Tabelas 13 e 14 adiante aonde as principais informações a respeito dos estudos estão dispostas sucedidas por uma discussão acerca das mesmas.

Examinando os estudos classificados no quadro acima tornou-se possível auferir uma inclinação dos pesquisadores em examinar o fenômeno das pequenas e médias empresas (PMEs) sob as lentes teóricas oferecidas pelas temáticas da RBV e internacionalização. Constatou-se que 30\% das pesquisas prestaram ênfase às organizações que fazem parte deste lócus de estudo. Posteriormente, notou-se que existe um interesse crescente nas pesquisas que envolvem as empresas denominadas de born globals, ou seja, aquelas que já nascem globais. Ademais, notou-se um interesse em elucidar o comportamento apresentado pelas organizações que decidem internacionalizar-se de economias emergentes para as desenvolvidas.

Destarte, de acordo com os delineamentos teóricos e metodológicos observados nos estudos, a contribuição da lente teórica da Visão Baseada em Recursos emerge - para os estudos que se dedicam a investigar os processos e trajetórias de internacionalização organizacional - na compreensão das diferenças específicas emanadas pelas empresas de posse de recursos distintos e, até mesmo, como se comportam estas organizações em busca de adquirir recursos estratégicos importantes. Estas diferenças se deram basicamente, nos casos aqui investigados, a nível comportamental e de adoções estratégicas

Tab. 13

Principais artigos em números de citações - WOS (2007 a 2016)

\begin{tabular}{|c|c|c|c|}
\hline № & Título / Autor / Periódico / Ano & Objetivo do Estudo & $\begin{array}{c}\# \\
\text { Citações }\end{array}$ \\
\hline 01 & $\begin{array}{l}\text { Title: What drives new ventures to internationalize from emerging to developed economies? } \\
\text { Author(s): Yamakawa, Yasuhiro; Peng, Mike W.; Deeds, David L. } \\
\text { Source: ENTREPRENEURSHIP THEORY AND PRACTICE Volume: } 32 \text { Published: Jan } 2008\end{array}$ & $\begin{array}{l}\text { Compreender o que impulsiona alguns novos } \\
\text { empreendimentos a se internacionalizarem de economias } \\
\text { emergentes para economias desenvolvidas. }\end{array}$ & 169 \\
\hline 02 & $\begin{array}{l}\text { Title: Conceptualizing accelerated internationalization in the born global firm: A dynamic } \\
\text { capabilities perspective. } \\
\text { Author(s): Weerawardena, Jay; Mort, Gillian Sullivan; Liesch, Peter W.; et al. } \\
\text { Source: JOURNAL OF WORLD BUSINESS Volume:42 Published: Sep } 2007\end{array}$ & $\begin{array}{l}\text { Apresentar um modelo conceitual do processo de } \\
\text { internacionalização acelerada de empresas nascidas globais. }\end{array}$ & 153 \\
\hline 03 & $\begin{array}{l}\text { Title: Alliance portfolio internationalization and firm performance. } \\
\text { Author(s): Lavie, Dovev; Miller, Stewart R. } \\
\text { Source: ORGANIZATION SCIENCE Volume: } 19 \text { Published: Jul/Aug } 2008\end{array}$ & $\begin{array}{l}\text { Introduzir a noção de internacionalização do portfólio de } \\
\text { alianças (API) - que se refere ao grau de estrangeirismo dos } \\
\text { parceiros na coleção de relações imediatas de alianças de uma } \\
\text { empresa - por meio do desenvolvimento de uma estrutura } \\
\text { para explicar como a API impacta o desempenho da empresa. }\end{array}$ & 112 \\
\hline 04 & $\begin{array}{l}\text { Title: Causes of the difficulties in internationalization } \\
\text { Author(s): Cuervo-Cazurra, Alvaro; Maloney, Mary M.; Manrakhan, Shalini } \\
\text { Source: JOURNAL OF INTERNATIONAL BUSINESS STUDIES Volume: } 38 \text { Published: Sep } 2007\end{array}$ & $\begin{array}{l}\text { Análise das causas das dificuldades enfrentadas pelas empresas } \\
\text { quando se internacionalizam em busca de novos mercados. }\end{array}$ & 107 \\
\hline 05 & $\begin{array}{l}\text { Title: The export orientation and export performance of high-technology SMEs in emerging } \\
\text { markets: The effects of knowledge transfer by returnee entrepreneurs. } \\
\text { Author(s): Filatotchev, Igor; Liu, Xiaohui; Buck, Trevor; et al. } \\
\text { Source: JOURNAL OF INTERNATIONAL BUSINESS STUDIES Volume: } 40 \text { Published: Aug } 2009\end{array}$ & $\begin{array}{l}\text { Examinar os fatores que afetam a orientação para exportação e } \\
\text { o desempenho das exportações de pequenas e médias } \\
\text { empresas (PMEs) de alta tecnologia em uma economia } \\
\text { emergente. }\end{array}$ & 104 \\
\hline 06 & $\begin{array}{l}\text { Title: Innovation practice and its performance implications in small and medium enterprises } \\
\text { (SMEs) in the manufacturing sector: A resource-based view. } \\
\text { Author(s): Terziovski, Mile } \\
\text { Source: STRATEGIC MANAGEMENT JOURNAL Volume: } 31 \text { Published: Aug } 2010\end{array}$ & $\begin{array}{l}\text { Identificar os impulsionadores da inovação e suas implicações } \\
\text { no desempenho das PMEs de manufatura. }\end{array}$ & 101 \\
\hline 07 & $\begin{array}{l}\text { Title: Third-World Copycats to Emerging Multinationals: Institutional Changes and } \\
\text { Organizational Transformation in the Indian Pharmaceutical Industry } \\
\text { Author(s): Chittoor, Raveendra; Sarkar, M. B.; Ray, Sougata; et al. } \\
\text { Source: ORGANIZATION SCIENCE Volume: } 20 \text { Published: Jan/Feb } 2009\end{array}$ & $\begin{array}{l}\text { Investigar como as empresas farmacêuticas indianas, } \\
\text { enfrentando mudanças institucionais descontínuas em seu } \\
\text { ambiente doméstico devido à liberalização econômica e às } \\
\text { reformas da propriedade intelectual, realizaram a } \\
\text { transformação organizacional. }\end{array}$ & 78 \\
\hline 08 & $\begin{array}{l}\text { Title: Toward a typology of commitment states among managers of born-global firms: A study } \\
\text { of accelerated internationalization } \\
\text { Author(s): Freeman, Susan; Cavusgil, S. Tamer } \\
\text { Source: JOURNAL OF INTERNATIONAL MARKETING Volume: } 15 \text { Published: } 2007\end{array}$ & $\begin{array}{l}\text { Identificar tipologias de estado de comprometimento dos } \\
\text { gestores de empresas nascidas globais com a } \\
\text { internacionalização acelerada das mesmas. }\end{array}$ & 74 \\
\hline 09 & $\begin{array}{l}\text { Title: The Role of Family Influence in Firms' Strategic Responses to Threat of Imitation } \\
\text { Author(s): Sirmon, David G.; Arregle, Jean-Luc; Hitt, Michael A.; et al. } \\
\text { Source: ENTREPRENEURSHIP THEORY AND PRACTICE Volume: } 32 \text { Published: Nov } 2008\end{array}$ & $\begin{array}{l}\text { Explicar o papel que a influência da família desempenha na } \\
\text { resposta às ameaças de imitação em empresas familiares. }\end{array}$ & 71 \\
\hline 10 & $\begin{array}{l}\text { Title: Internationalization of small and medium-sized enterprises (SMES) and international } \\
\text { entrepreneurship: A critique and policy implications } \\
\text { Author(s): Wright, Mike; Westhead, Paul; Ucbasaran, Deniz } \\
\text { Source: REGIONAL STUDIES Volume: } 41 \text { Published: } 2007\end{array}$ & $\begin{array}{l}\text { Estender a análise por meio da exploração de implicações } \\
\text { políticas associadas a recentes desenvolvimentos conceituais e } \\
\text { empíricos relacionados à internacionalização de PMEs. }\end{array}$ & 71 \\
\hline
\end{tabular}

Fonte: Web of Science (Fev./2017) 
mediante o intento da internacionalização dos negócios.

Contrastando com a situação encontrada nos dez artigos mais citados na base Web of Science, 0 enfoque prestado pelos autores dos trabalhos com maior número de citações na base Scopus mostrouse mais disperso mediante a abrangência de distintas perspectivas, não apresentando uma consonância significativa entre as temáticas. Foi possível notar que os pesquisadores associaram as temáticas da RBV e internacionalização também com o fenômeno bastante emergente das pequenas e médias empresas (PMEs) e a sua expressiva participação na economia global, estendendo suas análises para outros quatro enfoques.

A atenção voltou-se para a compreensão da performance (desempenho) internacional das organizações, fenômeno das born globals, empresas de países emergentes (EMEs) e redes. Ambos enfoques se encontraram suportados e investigados utilizando-se dos ditames teóricos da RBV, assim como propunha inicialmente o objetivo de investigação deste estudo ao compreender a contribuição da mesma para o estudo da internacionalização.

Tecendo uma análise geral dos quadros acima apresentados, evidenciou-se que o artigo mais citado na base WOS, com 169 citações, foi o "What drives new ventures to internationalize from emerging to developed economies?" com autoria de Yasuhiro Yamakawa, Mike W. Peng e David L. Deeds publicado em 2008 no Entrepreneurship Theory And Practice. 0 artigo trata da lacuna existente na literatura no que se refere a internacionalização de novos empreendimentos de economias emergentes para desenvolvidas buscando elucidar os impulsionadores que conduzem estes empreendimentos por meio da elaboração de uma tabela baseada em três perspectivas teóricas: a visão baseada na indústria, baseada em recursos e baseada na instituição.

Tab. 14

Principais artigos em números de citações - WOS (2007 a 2016)

\begin{tabular}{|c|c|c|c|}
\hline № & Título / Autor / Periódico / Ano & Objetivo do Estudo & $\begin{array}{c}\# \\
\text { Citações }\end{array}$ \\
\hline 01 & $\begin{array}{l}\text { Title: International business competence and the contemporary firm } \\
\text { Author(s): Knight, G.A., Kim, D. } \\
\text { Source: JOURNAL OF INTERNATIONAL BUSINESS STUDIES Volume:40 Published: } 2009\end{array}$ & $\begin{array}{l}\text { Investigar a internacionalização generalizada de pequenas e } \\
\text { médias empresas (PMEs) e os fatores específicos que } \\
\text { suportam seu desempenho superior no exterior. }\end{array}$ & 98 \\
\hline 02 & $\begin{array}{l}\text { Title: The role of family influence in firms' strategic responses to threat of imitation } \\
\text { Author(s): Sirmon, D.G., Arregle, J.-L., Hitt, M.A., Webb, J.W. } \\
\text { Source: ENTREPRENEURSHIP: THEORY AND PRACTICE Volume: } 32 \text { Published: } 2008\end{array}$ & $\begin{array}{l}\text { Explicar o papel que a influência da família desempenha na } \\
\text { resposta às ameaças de imitação em empresas familiares. }\end{array}$ & 98 \\
\hline 03 & $\begin{array}{l}\text { Title: Toward a typology of commitment states among managers of born-global firms: A } \\
\text { study of accelerated internationalization } \\
\text { Author(s): Freeman, S., Cavusgil, S.T. } \\
\text { Source: JOURNAL OF INTERNATIONAL MARKETING Volume: } 15 \text { Published: } 2007\end{array}$ & $\begin{array}{l}\text { Identificar tipologias de estado de comprometimento dos } \\
\text { gestores de empresas nascidas globais com a } \\
\text { internacionalização acelerada das mesmas. }\end{array}$ & 92 \\
\hline 04 & $\begin{array}{l}\text { Title: Capabilities as a mediator linking resources and the international performance of } \\
\text { entrepreneurial firms in an emerging economy } \\
\text { Author(s): Lu, Y., Zhou, L., Bruton, G., Li, W. } \\
\text { Source: JOURNAL OF INTERNATIONAL BUSINESS STUDIES Volume: } 41 \text { Published: } 2010\end{array}$ & $\begin{array}{l}\text { Investigar as relações entre capacidades, recursos e } \\
\text { desempenho internacional entre empresas empreendedoras } \\
\text { em uma economia emergente. }\end{array}$ & 90 \\
\hline 05 & $\begin{array}{l}\text { Title: Exploring the role of government involvement in outward FDI from emerging } \\
\text { economies } \\
\text { Author(s): Wang, C., Hong, J., Kafouros, M., Wright, M. } \\
\text { Source: JOURNAL OF INTERNATIONAL BUSINESS STUDIES Volume: } 43 \text { Published: } 2012\end{array}$ & $\begin{array}{l}\text { Explicar os mecanismos pelos quais o governo impacta a } \\
\text { internacionalização de empresas de países emergentes } \\
\text { (EMEs). }\end{array}$ & 75 \\
\hline 06 & $\begin{array}{l}\text { Title: Internationalization and performance: The moderating effects of organizational } \\
\text { learning } \\
\text { Author(s): Hsu, C.-C., Pereira, A. } \\
\text { Source: OMEGA Volume:36 Published: } 2008\end{array}$ & $\begin{array}{l}\text { a) Examinar o elo entre os recursos, a internacionalização e o } \\
\text { desempenho; } \\
\text { b) Investigar o papel moderador da aprendizagem } \\
\text { organizacional no elo internacionalização-desempenho. }\end{array}$ & 58 \\
\hline 07 & $\begin{array}{l}\text { Title: A comparative analysis of the internationalization of Chinese and Japanese firms } \\
\text { Author(s): Yang, X., Jiang, Y., Kang, R., Ke, Y. } \\
\text { Source: ASIA PACIFIC JOURNAL OF MANAGEMENT Volume: } 26 \text { Published: } 2009\end{array}$ & $\begin{array}{l}\text { Integrar visões de internacionalização de instituições, setores } \\
\text { e recursos e demonstrar que características industriais, } \\
\text { recursos empresariais e fatores institucionais podem explicar } \\
\text { as diferenças e semelhanças da expansão internacional de } \\
\text { empresas multinacionais chinesas e japonesas. }\end{array}$ & 57 \\
\hline 08 & $\begin{array}{l}\text { Title: Environmental strategy and exports in medium, small and micro-enterprises } \\
\text { Author(s): Martín-Tapia, I., Aragón-Correa, J.A., Rueda-Manzanares, A. } \\
\text { Source: JOURNAL OF WORLD BUSINESS Volume: } 45 \text { Published: } 2010\end{array}$ & $\begin{array}{l}\text { Analisar se as estratégias de proteção ambiental podem } \\
\text { ajudar pequenas e médias empresas (PMEs) à medida que } \\
\text { internacionalizam suas atividades por meio de exportações. }\end{array}$ & 51 \\
\hline 09 & $\begin{array}{l}\text { Title: Internalisation of ISO } 9000 \text { standards: The antecedent role of functionalist and } \\
\text { institutionalist drivers and performance implications } \\
\text { Author(s): Nair, A., Prajogo, D. } \\
\text { Source: INTERNATIONAL JOURNAL OF PRODUCTION RESEARCH Volume: } 47 \text { Published: } 2009\end{array}$ & $\begin{array}{l}\text { Investigar a relação entre a motivação para a certificação ISO } \\
9000 \text { e a internalização de práticas subjacentes às normas } \\
\text { ISO } 9000 .\end{array}$ & 51 \\
\hline 10 & $\begin{array}{l}\text { Title: Network resource combinations in the international venturing of small biotech firms } \\
\text { Author(s): Tolstoy, D., Agndal, H. } \\
\text { Source: TECHNOVATION Volume: } 30 \text { Published: } 2010\end{array}$ & $\begin{array}{l}\text { Analisar como pequenas empresas de biotecnologia } \\
\text { comercializam inovações combinando recursos em suas } \\
\text { redes para entrar em novos mercados estrangeiros com } \\
\text { produtos existentes e entrar nos mercados estrangeiros } \\
\text { existentes com novos produtos. }\end{array}$ & 50 \\
\hline
\end{tabular}

Fonte: Web of Science (Fev./2017) 
Já na Scopus, o artigo que se sobressaiu com 98 citações foi o "International business competence and the contemporary firm" dos autores Gary A. Knight e Daekwan Kim publicado em 2009 no periódico Journal of International Business Studies. O trabalho dedicou-se a tratar da internacionalização das pequenas e médias empresas (PME's) objetivando investigar os aspectos gerais e específicos que conferem um desempenho superior internacional às PME's na medida em que estas contam com muito menos recursos financeiros $e$ tangíveis do que as grandes empresas multinacionais. Como resultado identificou-se a competência comercial internacional (IBC) como um dos recursos geradores de desempenho superior para as PME's. Ademais, um modelo que relacionasse a IBC com o desempenho internacional das pequenas e médias empresas foi desenvolvido e avaliado.

Por meio desta análise, notou-se que dois artigos figuraram como mais citados em ambas as bases de dados, modificando apenas a colocação destes quanto ao número de citações. Para fins de verificação, os mesmos se encontram em destaque nas Tabelas 13 e 14. Ademais, percebe-se que em ambas as bases de dados, a grande maioria dos autores das publicações mais citadas não estão entre os autores que mais publicaram no período. A única exceção foi o autor Mike Wright que figurou na 1a posição no ranking dos que mais publicaram no período na base WOS e cujo artigo aparece dentre os que mais foram citados, ocupando a posição 5 na Scopus e 10 na WOS.

\section{CONCLUSÃO}

O presente estudo contou com o objetivo de verificar a contribuição da Visão baseada em recursos na Internacionalização de empresas por meio de um estudo bibliométrico que buscou analisar as características das publicações sobre as temáticas, nas bases de dados Web of Science e Scopus, no período de 2007 a 2016, e identificar quais tópicos estão sendo estudados junto a este tema e aqueles que constituem-se nos mais relevantes (hot topics), realizando uma comparação dos resultados encontrados nas duas bases de dados (WOS e Scopus).

Neste sentido, constatou-se por meio do estudo que o tema Visão baseada em recursos e Internacionalização se encontra em evolução no que tange o número de publicações. Apesar de não apresentar crescimento constante nos últimos dez anos, a temática alcançou seu pico de publicações no ano de 2016, conforme a base WOS. Entre os periódicos com maior número de publicações na base WOS, destaca-se o International Business Review e na base Scopus o Journal of World Business.

Os Estados Unidos lideram o ranking dos países que mais publicam sobre a temática nas duas bases. Na WOS a Inglaterra é o segundo país que mais publica, seguido por Espanha, China e Austrália. $\mathrm{Na}$ Scopus, o Reino Unido é o segundo que mais publica, seguido por Austrália, Espanha, Brasil e China. Foi possível, ainda, classificar como hot topics, duas combinações da temática na base Web of Science: Performance (desempenho) e Strategy (estratégia).

O estudo possibilitou a construção de dois quadros com as dez publicações mais citadas em cada uma das bases aonde realizou-se uma comparação com os autores que mais publicaram no período pesquisado, elucidando que as publicações com o maior número de citações não pertencem aos autores que mais publicam sobre o mesmo tema no período determinado para a pesquisa (2007-2016), exceto no caso do autor Mike Wright.

Durante a realização do trabalho, tornou-se possível reconhecer e salientar a utilidade que os mecanismos de busca possuem para as pesquisas acadêmicas. A utilização de bases como a WOS e a Scopus, serve de ferramenta para o desenvolvimento das pesquisas por meio do acesso possibilitado à comunidade acadêmica no que tange às publicações e informações a respeito da evolução dos temas de interesse dos pesquisadores. A relevância deste trabalho reside na compilação das características fundamentais das publicações que integram o referido campo teórico investigado o que pode favorecer o surgimento de insights para pesquisas futuras.

Por conseguinte, as limitações que podem ser apontadas para o presente estudo se referem ao levantamento ter sido realizado junto a duas bases de dados específicas (WOS e Scopus). Ademais, recomenda-se que estudos futuros de mesma natureza ampliem a busca para outras bases e busquem verificar também, o cenário nacional da temática utilizando-se de eventos acadêmicos nacionais e periódicos com notável expressão no campo de estudo. 


\section{REFERENCES}

- $\quad$ ARAújO, C. A. (2006). Bibliometria: Evolução Histórica e Questões Atuais. Em Questão, v. 12, n. 1, p. 11- 32.

- $\quad$ AVILA, L. V.; BARROS, I. C. F.; MADRUGA, L. R. R. G.; JÚNIOR, V. F. S. (2014). Características das publicações sobre Empreendedorismo (Social) no Web of Science no período 2002-2011. Administração Pública e Gestão Social, v.6(2), abr-jun, p.88-100.

- BANKS, M. G. (2006). An extension of the hirsch index: indexing scientific topics and compounds. Recuperado de: <http://www.arxiv.org/abs/physics/0604216>. Acesso em: fevereiro 2017.

- BARNEY, J. B. (1986). Organizational culture: Can it be a source of sustained competitive advantage? Academy of Management Review, v. 11.

- (1986). Strategic factor markets: expectations, luck and business strategy. Management Science, vol. 32, n. 10, p.1231-1241. (1991). Firm resources and sustained competitive advantage. Journal of Management, vol. 17, p. 99-120.

- CAPES - COORDENAÇÃO DE APERFEIÇOAMENTO DE PESSOAL DE NÍVEL SUPERIOR. Acervo. 2017. Disponível em: <http://www-periodicos-capes-govbr.ez47.periodicos.capes.gov.br/index.php?option=co m_pcollection\&mn=70\&smn=79\&cid $=81 \& \mid$ temid $=\&>$. Acesso em: Fev. 2017.

- CARPES, A. de. M.; SCHERER, F. L.; VELTER, A. N; LÜTZ, C. (2011). Panorama Internacional das Publicações em International Business: Levantamento através da Base Web of Science. In: Encontro da ANPAD, XXXV EnANPAD, Rio de Janeiro. Anais... Rio de Janeiro: EnANPAD.

- CHUEKE, G.V.; AMATUCCI, M. (2015) O que é bibliometria? Uma introdução ao Fórum. Revista Eletrônica de Negócios Internacionais (Internext), v.10, n. 2, p. 1-5, mai./ago. São Paulo.

- CRONIN, B. (2001) Bibliometrics and beyond: some thoughts on web-based citation analysis. Journal of Information Science, 27 (1), p. 1-7.

- DhANARAJ, C.; BEAMISH, P. W. (2003). A Resourcebased approach to the study of export performance. Journal of Small Business Management, Milwaukee, v. 41, n.3, p.242-261, July.

- DUNNING, J. H. (1980) Toward an Eclectic Theory of International Production: Some Empirical Tests. Journal of International Business Studies, v. 11, n. 1, p. 9-31.

- FAHY, J. (2002). A resource-based analysis of sustainable competitive advantage in a global environment. International Business Review, v.11, p.5778.

- FAVORETO, R. L.; AMÂNCIO-VIEIRA, S. F.; SHIMAdA, A. P. (2014) A produção intelectual em RBV: Uma incursão bibliométrica nos principais periódicos internacionais.
Revista Brasileira de Estratégia, v. 7, n. 1, p. 37-55, jan/mar.

- GRANT, R. M. (1991). The resource-based theory of competitive advantage: Implications for strategy formulation. California Management Review, v. 33, n. 3, Spring.

- HANSEN, M. H.; PERRY, L. T.; REESE, C. S. (2004). A Bayesian operationalization of the resource-based view. Strategic Management Journal, v. 25, Issue 13.

- HIRSCH, J. E. (2005). An index to quantify an individual's scientific research output. Proceedings of the National Academy of Sciences of the United States of America.

- $\quad$ HITT, M. A.; BIERMAN, L.; UHLENBRUCK, K.; SHIMIZU, K. (2006) The importance of resources in the internationalization of professional service firms: The good, the bad, and the ugly. Academy of Management Journal, 49, p. 1137-1157.

- JOHANSON, J.; WIEDERSHEIM-PAUL, F. (1975). The internationalization of the firm: Four Swedish cases. Journal of Management Studies, Blacwell Publishing, Oxford and Malden, v. 12, n. 3, p. 305-322.

- $\quad \mathrm{KNIGHT}$, G. A.; KIM, D. (2009). International business competence and the contemporary firm. Journal of International Business Studies, v. 40, n. 2, p. 255-273.

- KRAAIJENBRINK, J.; SPENDER, J. C.; GROEN, J. A. (2010). The Resource-Based View: A Review and Assessment of Its Critiques. Journal of Management. v. 36.

- MORAES, S. G.; STREHLAU, V. I.; TUROLLA, F. A. (2015) Produção acadêmica de autores brasileiros sobre Internacionalização: Balanço das publicações no Brasil no Séc. XXI. Revista Eletrônica de Negócios Internacionais (Internext), v.10, n. 2, p. 82-96, mai./ago. São Paulo.

- MOTKE, F. D.; RAVANELLO, F. S.; RODRIGUES, G. O. (2016) Teoria Institucional: Um Estudo Bibliométrico da Última Década na Web of Science. Contextus - Revista Contemporânea de Economia e Gestão, v. 14, p. 63.

- NEWBERT, S. L. (2007). Empirical research on the resource-based view of the firm: an assessment and suggestions for future research. Strategic Management Journal, v.28, Issue 2 .

(2008). Value, rareness,

competitive advantage, and performance: a conceptual level empirical investigation of the resource-based view of the firm. Strategic Management Journal, v.29, Issue 7.

- PENG, M. W. (2001). The resource-based view and international business. Journal of Management, v. 27, n. 6, p. 803-829.

- PENROSE, E. T. (1959). The theory of the growth of the firm. New York: John Wiley.

- Peteraf, M. A. (1993). The cornerstones of competitive advantage: a resource-based view. Strategic Management Journal, v. 14. 
- PRAHALAD, C. K.; HAMEL, G. (1990). The core competence of the Corporation. Harvard Business Review, may-june, p. 79-91.

- REID, S. D. (1981). The decision-maker and export entry and expansion. Journal of International Business Studies, 12 (Fall): 101-12.

- ROCHA, A. C.; CAMARGO, C. R.; KNEIPP, J. M.; GOMES, C. M.; ZAMBERLAN, J. F. (2013) Recursos Hídricos e Gestão: um estudo bibliométrico da produção científica e dos hot topics publicados na base Web of Science na última década. Espacios (Caracas), v. 34, p. 6.

- $\quad$ SAlAZAR, V. S.; MORAES, W. F. A. de.; LeITE, Y. V. P. (2011) Resource based view: Das proposições basilares de Penrose à internacionalização das empresas contemporâneas. In: Encontro Nacional de Engenharia de Produção, XXXI ENEGEP, Belo Horizonte, 2011. Anais... Belo Horizonte: ENEGEP.

- SANCHO, R. (2002) Indicadores bibliométricos utilizados en la evaluación de la ciencia y la tecnologia: revisión bibliográfica. In Inteligencia competitiva: documentos de lecture. [Em linha]. Barcelona: Fundació per a la Universitat Oberta de Catalunya, p.77-106. Disponível em: <

http://digital.csic.es/bitstream/10261/23694/1/SAD_DI G_IEDCyT_Sancho_Revista\%20Espa\%C3\%B1ola\%20de \%20Documentacion\%20Cientifica13\%284\%29.pdf

- SHARMA, V. M.; ERRAMILLI, M. K. (2004). Resourcebased explanation of entry mode choice. Journal of Marketing Theory and Practice, v. 12, n. 1, p. 1-18, Winter.
- $\quad$ SOUZA, E. C. L. de.; FENILI, R. R. (2012).

Internacionalização de empresas: Perspectivas teóricas e agenda de pesquisa. Revista de Ciências da Administração, v.14, n. 33, p. 103-118, ago.

- VANTI, N. A. P. (2002). Da bibliometria à webometria: uma exploração conceitual dos mecanismos utilizados para medir o registro da informação e a difusão do conhecimento. Ciência da Informação, v. 31, n. 2, p. 152-162, maio/ago.

- VERNON, R. (1966) International Investment and International Trade in the Product Cycle. The Quarterly Journal of Economics, v. 80, n. 2, p. 190-207.

- ZEN, A. C.; FENSTERSEIFER, J. E.; PRÉVOT, F. (2014). O impacto dos recursos do desempenho exportador de empresas pertencentes a clusters: Um estudo no setor vitivinícola francês. R. bras. Gest. Neg., São Paulo, v.16, n.52, p. 374-391, jul./set.

$\cdot$

(2009). A Internacionalização de Empresas em Clusters Industriais e a Visão Baseada em Recursos. In: Encontro da Associação Nacional dos Programas de PósGraduação Em Administração, XXXIII EnANPAD, São Paulo, 2009. Anais... São Paulo: ANPAD.

- WeRnerfelt, B. (1984). The Resource-Based View of the Firm. Strategic Management Journal, n. 5, p. $171-$ 180.

- WESTHEAD, P; WRIGHT, M.; UCBASARAN, D. (2001) The internationalization of new and small firms: A resourcebased view. Journal of Business Venturing, 16, p. 333358.

\section{SOBRE AS AUTORAS}

- Maíra Nunes Piveta é Mestranda em Administração do Programa de Pós Graduação em Administração da UFSM. E-mail: mairanpiveta@gmail.com

- Flavia Luciane é Doutora em Administração pela UFMG e Professora no Departamento de Ciências Administrativas da UFSM. E-mail: flaviascherer@globo.com

- Aletéia de Moura Carpes é Doutora em Administração pela UFSM e professora de Administração na UNIFRA E-mail: alecarpes.adm@gmail.com

- Nathália Rigui Trindade é Mestranda em Administração do Programa de Pós Graduação em Administração da UFSM. E-mail: nathaliariguitrindade@gmail.com

- Andréa Bach Rizzatti é Mestranda em Administração do Programa de Pós Graduação em Administração da UFSM E-mail: andrea-bach@hotmail.com

- Maríndia Brachak dos Santos é Doutoranda em Administração do Programa de Pós Graduação em Administração da UFSM e professora no Colégio Politécnico da UFSM E-mail: marindiabrachak@gmail.com 


\title{
The contribution of the resource-based view to the internationalization study: a bibliometric analysis of scientific production between 2007 and 2016
}

Maíra Nunes PivetaA, Flavia Luciane Scherer ${ }^{A}$, Aletéia de Moura Carpes ${ }^{B}$, Nathália Rigui Trindade ${ }^{A}$, Andréa Bach Rizzatti ${ }^{A}$ e Maríndia Brachak dos Santos ${ }^{A}$

${ }^{A}$ Universidade Federal de Santa Maria, UFSM, Santa Maria, RS, Brasil.

${ }^{B}$ Centro Universitário Franciscana, UNIFRA, Santa Maria, RS, Brasil.

ARTICLE DETAILS
Article history:
Received: 31 August 2017
Reviewed: 17 November 2017
Accepted: 04 February 2018
Available online: 01 May 2018
Double Blind Review System
Scientific Editor
llan Avrichir
Keywords:
Resource Based View
Internationalization
Web of Science
Scopus
Bibliometrics

\begin{abstract}
This study aimed to analyze the characteristics of publications patterns based on the Resource Based View (RBV) and Internationalization, in the databases Web of Science and Scopus, from 2007 to 2016, and to identify the topics related with this study and which of them are the most relevant (hot topics), making a comparison of the results found in the two databases. It has a quantitative approach considering that it sought to quantify some variables referring to the scientific production on the researched topic. Searches for publications on VBR and Internationalization in the period from 2007 to 2016 found 210 publications in the WOS database and 127 in Scopus. By comparing the bases surveyed, it was verified that the number of publications grew in the last two years and that the United States leads the ranking in the number of publications. It is noticed that in both databases, the authors of the most cited publications are not among the authors who published most in the period, except for Mike Wright. Two combinations were classified as hot topics in the WOS: Performance and Strategy.
\end{abstract}

(C) 2018 Internext | ESPM. All rights reserved!

Para citar este artigo:

Piveta, M. N., Scherer, F. L., Carpes, A. de M., Trindade, N. R., Rizzatti, A. B., e Santos, M. B. (2018) A contribuição da visão baseada em recursos para o estudo da internacionalização: uma análise bibliométrica da produção científica entre os anos de 2007 e 2016. Internext - Revista Eletrônica de Negócios Internacionais, 13 (2), 43-58. DOI: http://dx.doi.org/10.18568/1980-4865.13243-58

Para acessar este artigo: http://dx.doi.org/10.18568/1980-4865.13243-58 